\title{
Gold-Decorated Nanodiamonds: Powerful Multifunctional Materials for Sensing, Imaging, Diagnostics, and Therapy
}

\author{
Silvia Orlanducci*[a]
}

6

tifunctional material that can find space in numerous applications in the biological field. The coupling and/or synergy of the properties of the individual components can offer interesting solutions in the field of optical labeling, imaging, molecule and drug delivery, and biosensing. This microreview describes the 11 techniques used for the synthesis of these materials and in- cludes a critical discussion of some recent results on their application. The synergy between the two particles is particularly evident in the improvement of the plasmonic and photoacoustic properties of gold. In addition, the catalytic and sensor activity of the hybrid system shows peculiarities not found in the individual components alone.

\section{Introduction}

The design of multifunctional nanoparticles for sustainable technological applications is nowadays one of the most promis-

21 ing research fields. Multifunctionality is an intriguing hot topic in materials science, the possibility to design nanoscale materials with tailored properties, and cooperative or hieratic functions open new technological scenario with a far-reaching range of applications. As an example, there is a considerable

26 interest in the development of nanoparticles capable of diagnosing and treating cancer at the same time or delivering and releasing specific molecules and drugs on demand.

Functionalized or decorated nanoparticles are typical multifunctional materials; thanks to the introduction of specific func-

31 tions and to the synergy between the two components, these systems provide new potential for theranostics applications. Several reviews described multifunctional nanoparticles: as versatile nanocarriers, ${ }^{[1]}$ target-specific magnetic particles, ${ }^{[2]}$ multifunctional nanoparticles for multimodal imaging, ${ }^{[3]}$ stimuli re36 sponsive nanoparticles. ${ }^{[4]}$ The investigated materials range from

[a] Department of Chemical Science and Technology, University of Rome "Tor Vergata",

Via della Ricerca Scientifica 00133 Roma, Italy

E-mail: silvia.orlanducci@uniroma2.it

口 ((<=Author: please insert the URL of your group or institute))

(D) $O R C I D(s)$ from the author(s) for this article is/are available on the WWW under https://doi.org/10.1002/ejic.201800793. liposomes, dendrimers, polymeric micelles, metal oxides, silica, gold nanoparticles, carbon nanomaterials that can be functionalized, decorated, mixed to each other to add specific polyvalence. In this large class of systems, we will focus on gold nanoparticles (AuNPs) and nanodiamonds (NDs) as well as on hybrid 41 nanoparticles (AuNP-ND) based on AuNPs and NDs, a recently developed multifunctional nanomaterial with promising applications in biological environments.

Gold nanoparticles and nanodiamonds are already individually precious materials proposed and investigated in many re- 46 search fields. Both of them detain incomparable electronic and physical properties, an easily modifiable surface chemistry and finally, they are biocompatible. These features have made gold nanoparticles and nanodiamond ones of the most widely used nanomaterials for academic research and proposed materials 51 for a broad range of applications. ${ }^{[5-8]}$

Colloidal gold nanoparticles have an ancient history; they have been used for the preparation of dichroic glass already during the Roman Empire ${ }^{[9]}$ and as an alchemical drug of longevity in traditional Indian and Chinese medicine as well as to 56 treat nervous disorders and syphilis in Europe. ${ }^{[10]}$ The typical ruby color of a colloidal gold nanoparticles solution arises from the resonance excitation of localized surface plasmons (LSP).

The main physical effects, moving from bulk Au to Au nanoparticles, are the formation of a maximum in the extinction 61 spectrum at the plasmon resonance frequency, and a great en-

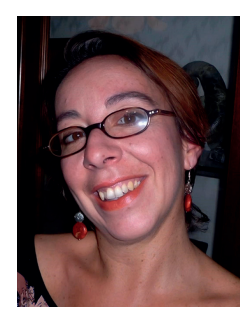

Silvia Orlanducci received her Ph.D. in Chemical Sciences in 2004. Since 2007 she has been a Researcher at the Faculty of Science of the Tor Vergata University of Rome (Italy), where she is presently Associate Professor. Her research activity, performed in the frame of Inorganic Chemistry and Material Chemistry, is focused on the synthesis methodologies, treatment, and structural/functional characterization of materials. Her main research line deals with carbon-based nanomaterials: nanodiamonds, nanotubes, nanographites, graphene, and carbon nanoparticles. 
hancement of electromagnetic field near to the nanoparticles itself. The extinction cross-section can be 1000 times larger than the geometrical size of particles and is dominated by absorp66 tion in the case of small particles $(<30 \mathrm{~nm})$ and by scattering for larger particles. LSP of gold nanoparticles can range from visible to NIR wavelengths, and depend on size, aspect ratio, shape, surface functionalization, aggregation state and dielectric properties of the environment. ${ }^{[11-13]}$

71 Several synthetic and functionalization procedures have progressed and now nanoparticles with selected size distribution and various shapes, such as sphere, rods, stars, shell, triangles and many others, are available. ${ }^{[14-16]}$

AuNPs are applied with remarkable results in many research 76 field including chemical and biological sensing, ${ }^{[17]}$ photocatalysis, $^{[18]}$ nano-optics, ${ }^{[19]}$ and living cell real-time surface enhanced Raman spectroscopy (SERS) and imaging. ${ }^{[20,21]}$

Nanodiamonds are a nanoscale diamond with particles size ranging between 4 to $100 \mathrm{~nm}$. Several synthesis techniques us-

81 ing both bottom-up and top-down approach have been developed. ${ }^{[8]}$

NDs can be obtained directly either by detonation method or laser pyrolysis. ${ }^{[2,23]}$ Secondly, they can be produced by milling of bulk diamond which could be natural diamond or dia86 mond grown by CVD or HPHT. ${ }^{[24,25]}$

Other methods include: the hydrothermal synthesis from carbides or from organic compounds, ${ }^{[26,27]}$ high energy electrons or ions irradiation of carbon materials ${ }^{[28,29]}$ and ultrasound cavitation. ${ }^{[30]}$

91 The most commonly used and commercially accessible nanodiamonds are those obtained by detonation or by milling of microsized diamond (HPHT or natural). Whereas, pulse laser synthesized ND powder became commercially available recently.

96 The nanodiamonds obtained by detonation and those by grinding of larger crystals show substantial differences in terms of structure, morphology and properties. The major difference is related to the size of primary particles that are in the range of 4-5 nm with spherical morphologies for detonation dia-

$101 \mathrm{mond}$, and around 10-20 nm with an irregular facet shape in the case of ND from fragmentation of larger diamonds.

Another important difference between the two kinds of nanodiamond is related to the photoluminescence properties. Nanodiamonds have some of the remarkable physical proper106 ties of diamonds including point defects, as vacancies and impurities, which are responsible for the fluorescence of diamonds. Fluorescence nanodiamonds are extremely photostable ensuring long-term photoluminescence without photobleaching, showing a brightness over two orders of magnitudes

111 higher than typical dye molecules moreover nitrogen vacancies (NV) and silicon vacancy (SiV), the most studied optical centers in diamond, emit in red and in near-infrared spectral region respectively therefore at wavelengths that easily penetrate biological tissue. ${ }^{[31-33]}$

116 ND from HPHT diamond are the preferred candidate for imaging application showing stable and intense fluorescence in particular after irradiation and annealing process. ${ }^{[25]}$ While instead detonation nanodiamonds are not able to host stable fluorescence defects mainly because the defects are too near the surface allowing preferentially a non-radiative decay. Many 121 types of research are still underway to obtain stable fluorescent diamonds smaller than $10 \mathrm{~nm}$ and in a larger amount. ${ }^{[34,35]}$

The nanodiamond retains most of the incredible properties of the diamond such as extremely high hardness, high wear resistance, high thermal conductivity, and electrical resistivity, 126 optical properties, chemical stability also in extreme environment and biocompatibility. It is important to underline that thermal properties of nanodiamond (thermal conductivity and thermal stability) as well as of all nanocrystalline materials, strongly depend on the size and surface termination. In particu- 131 lar, thermal conductivity is both governed by the properties of the grain boundaries and by the grain size and strongly decrease with the lowering of the size of crystals. ${ }^{[36]}$

Nevertheless, the interface conductance (Kapitza conductance) of nanodiamond seem to be ten times larger than that 136 reported for any other material[ ${ }^{[37]}$ confirming the useful role of nanodiamond as excellent heat dissipation filler material in nanocomposites.

Hydrogen terminated nanodiamonds, as well as diamonds, exhibit negative electron affinity (NEA), which strongly im- 141 proves the field emission and the photoemission from the nanodiamond surface. The generation of electrons can be used in cold cathode technology ${ }^{[38]}$ but also in the generation of solvated electrons and radicals for photocatalysis ${ }^{[39,40]}$ or reactive oxygen species for in-vitro treatment of radioresistant cancer 146 cells. ${ }^{[41]}$

Compare to diamond, ND are characterized by high surface to volume ratio, and their chemical-physical properties are strongly related to the chemical groups on their surface. Several reviews describe well-developed methods for modifying the 151 surface termination of nanodiamonds made from different technologies. ${ }^{[42,8]}$ Nanodiamonds surface can be easily manipulated by covalent and not covalent functionalization with organometallics, polymers, drugs, DNA, RNA, proteins, peptides, and many others. ${ }^{[43,44]}$

Today small NDs (4-10 nm) are synthesized in large quantities at a relatively low cost by detonation methodology and are the most frequently experimented in the biological and medical application. In vitro and in vivo animal studies, including oral, subcutaneous, intramuscular and intravenous administration, 161 demonstrated the absence of genotoxicity cytotoxicity, biocompatibility, and non-toxicity of nanodiamonds. ${ }^{[45-49]}$

Considering the unique and complementary properties of the two nanomaterials, it is not surprising that some research groups are evaluating the potentials expressed by the 166 AuNP-ND hybrid system.

\section{Synthesis and Characterization of AuNP-ND Hybrid Nanoparticles}

The control of the shape and size of gold nanoparticles on nanodiamond surface, as well as the functionalization of the ND 171 surface, and the mutual organization between the two types of nanoparticles represent the key steps to define the ultimate use of the systems. Two general synthesis procedures have been 
proposed: the direct synthesis of gold nanoparticles in a nano-

176 diamond dispersion and the post-synthesis fastening of AuNPs on ND surface by covalent or non-covalent bonds. The simplest way to prepare the AuNP decorated ND is the direct reduction of gold ions on nanodiamonds surface by means of reducing agents as $\mathrm{NaBH}_{4}{ }^{[50-53]}$ or hydroxylamine hydrochloride. ${ }^{[54]}$ The

181 reducing agent can be replaced by introducing proper chemical groups directly on nanodiamond surface able to reduce gold ions. ${ }^{[55]}$ Hydroxyl groups on nanodiamond play the principal role as reducing agent; they are always present in a certain quantity on the surface of purified hydrophilic nanodiamonds

186 but can be further introduced on nanodiamond surface by reduction of carboxyl groups by boranes ${ }^{[42]}$ or by radical addiction as in Fenton protocol. ${ }^{[56]}$ This protocol uses hydrogen peroxide $\mathrm{H}_{2} \mathrm{O}_{2}$ and iron sulfate $\mathrm{FeSO}_{4}$ in a strongly acidic solution and is able to introduce $\mathrm{OH}$ groups by radical addiction and

191 clear the surface from non-diamond carbon.

Adding reducing agent directly on ND surface itself could be an elegant procedure that significantly reduces the consumption of chemicals and shortens the purification processes duration.

196 The direct synthesis of AuNPs in a NDs dispersion generally leads to growth spherical or quasi-spherical particles while synthesis duration and conditions (mainly temperature and $\mathrm{pH}$ ) have an effect on particle size distribution.

Quasi-ellipsoidal nanoplatelets, Figure 1, were synthesized by

201 Matassa et al. ${ }^{[57]}$ using detonation nanodiamond $\mathrm{OH}$ functionalized and an innovative Au complex. ${ }^{[58]}$ Under the experimental parameters, AuNPs show a discoidal shape having a diameter of about $30 \mathrm{~nm}$, and a thickness of $10 \mathrm{~nm}$, moreover, ND particles are sometimes embedded into the Au platelets. Such

206 unusual assembling could be related to a selective interaction of $\mathrm{Au}$ complex with the ND surfaces driving the synthesis of this hybrid AuNP-ND nanomaterial.

Ones of the advantages of this procedure is the simplicity of methodology, the long-term stability of the dispersion, and the 211 intimate contact between the two species: gold and diamond.

AuNPs with more controlled shape and size distribution have to be previously synthesized and then attached to nanodiamond by post synthesis protocols.

Yun Zeng et al. ${ }^{[59]}$ use sophisticated methods to decorate 216 nanodiamond with gold nanorods. They use polydopamine (PDA) coated ND, as a platform to further modifications, and DNA functionalized gold nanorods (AuNR). After bonding DNA strand by tiolate bond on PDA-ND, the two systems bind to each other by the complementary hybridization of DNA strands

221 on both nanoparticles. An example of DNA mediated selfassembly of AuNR with ND (AuNR-ND) structures are reported in Figure 2.

$\mathrm{Au} / \mathrm{Ag}$ urchin-like NPs were conjugated to detonation ND by thiol bond as reported in Ref 60. Urchin-like or stars-like AuNPs

226 are multi-branched particles showing a strong electromagnetic field enhancement due to the sharp edges of the tips. Also, in this case, the synthetic procedure consists in many steps starting with the covalently bonding of ND with poly(ethylene glycol) bis(3-aminopropyl) terminated (PEG1500N) that intro231 duce a broad fluorescence by passivation of ND surface (giving
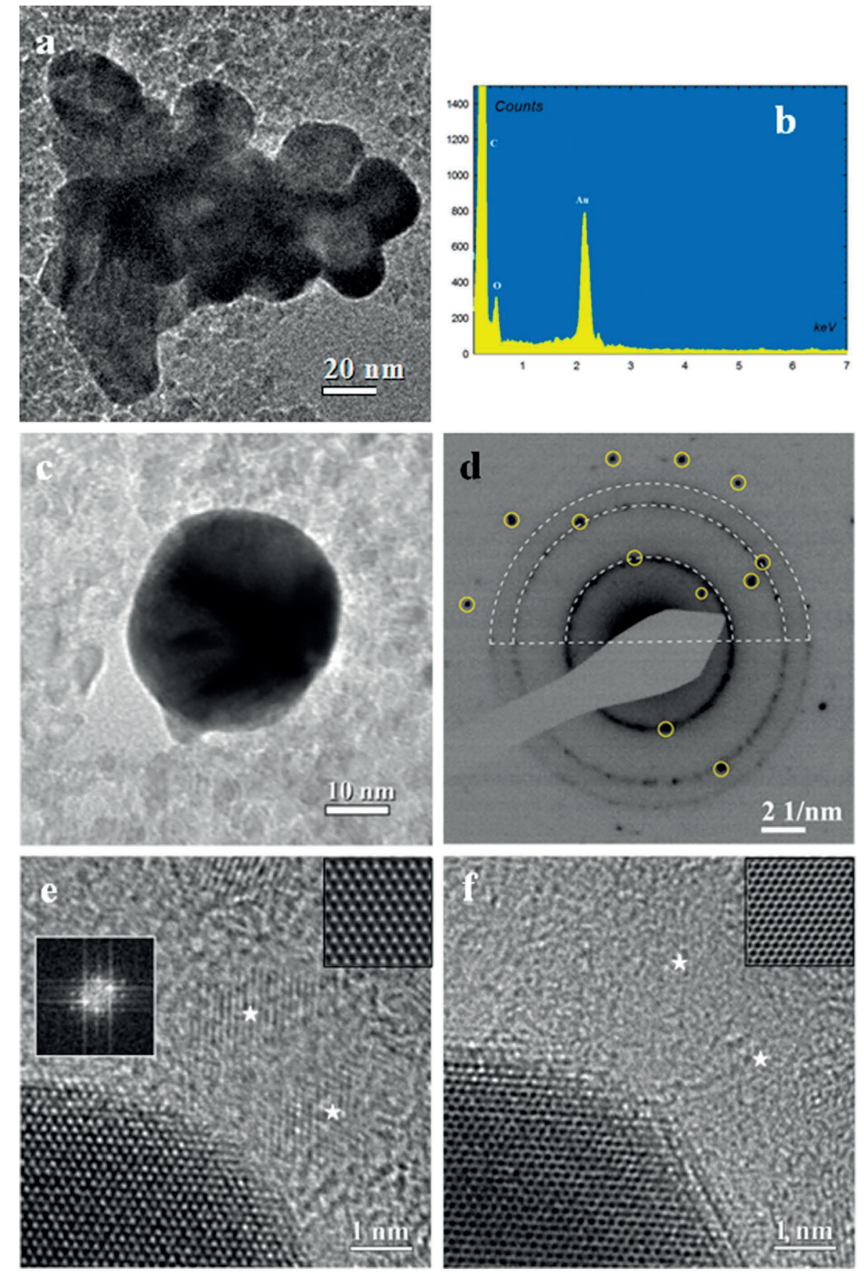

Figure 1. Morphology and structural studies of AuNP-ND nanostructures. TEM image of AuNP-ND nanoplatelet aggregates (a) and of a single nanoplatelet (c). (b) EDX spectral image; the peak at about $2 \mathrm{keV}$ is the Au signal. (d) Electron diffraction pattern of the ND phase (white dot arcs) and the fcc Au phase (yellow circles). (e) and (f) HR TEM images at the edge of an Au nanoplatelet; NDs are labeled by white stars. Reprinted with permission from ref. $^{[57]}$ Creative Commons Attribution 4.0 International License.

fluorescent nanodiamond FND). Then, using a reagent able to react selectively with the primary amine of PEG1500N, thiolated nanodiamonds were prepared and finally, were conjugated with $\mathrm{Au} / \mathrm{Ag}$ NPs. Another attractive protocol to decor nanodiamond with gold nanoparticles are developed by W. Liu et al. ${ }^{[61]}$ Here 236 the hybrid particles were prepared by absorption and crosslinking of controlled protein coated AuNPs on carboxylated ND. Finally, by applying ultracentrifugation procedures AuNP-ND dispersions with narrow size distributions and long-term stability were obtained.

\section{Multifunctional Applications of AuNP-ND Hybrid Nanoparticles}

\subsection{AuNP-NDs as Imaging Agents}

The use of AuNP-ND hybrid system was very effective in photoacoustic tomography. In analogy with the graphene used as 246 

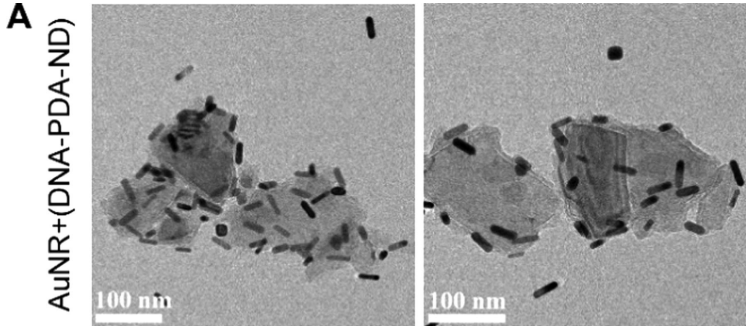

B

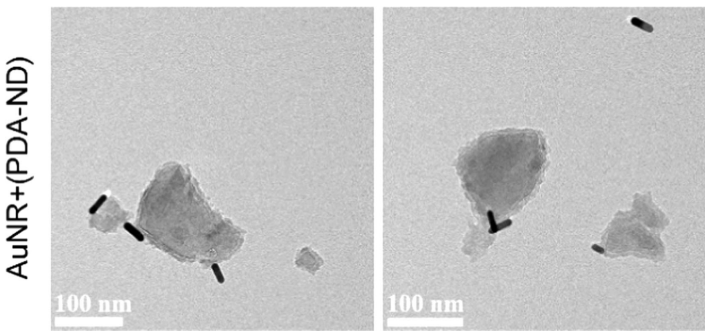

Figure 2. TEM images of: (A) AuNR-NDs via specific DNA hybridization; (B) mixture of PDA-ND and AuNRs without specific DNA hybridization. Reprinted with permission from ref. ${ }^{[59]}$ Copyright 2018 American Chemical Society. tween the excited nanodiamond and the AuNP capable to support this remarkable amplification. ${ }^{[63]}$

Instead, in the case of detonation $\mathrm{ND}^{[51]}$ the authors perform in vivo experiment using NIR sources, that is more suitable for future tomographic applications of these systems, and in addition to signal amplification, they highlight a longer lifetime of the AuNPs, Figure 4.

This effect is due to the greater resistance (durability) of the gold nanoparticles, in fact, under irradiation the developed heat can be easily transferred from gold to the diamond and then dissipates it in the medium, slowing down the dissolution of the metal. Finally, the authors report cytotoxicity studies high- 276 lighting the low toxicity of the system even at high contractions (125 ug/mL).

ND gold and silver nanoparticles are highly efficient in photothermal therapy against cancer cells as proved by Liang-Chien Cheng et al. ${ }^{[60]}$ In this paper, the authors described the prepara- 281 tion of urchin-like Au/Ag NPs conjugated with ND and tested this system as photothermal therapeutic material under NIR irradiation. A great advantage was relieved by labeling the nanoparticles by human transferrin (Tf) leading both an increase of
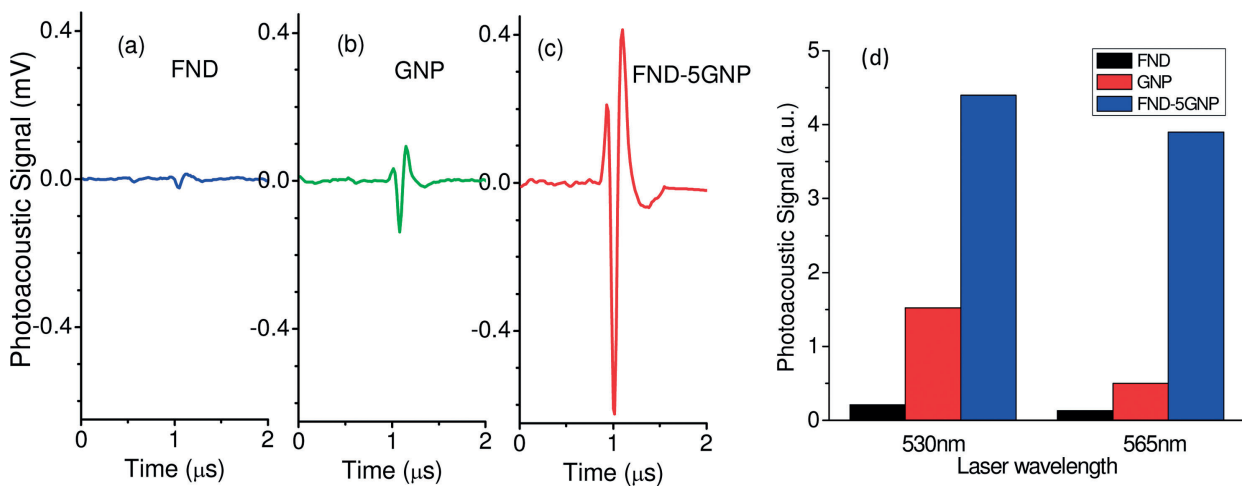

Figure 3. In-silicon PA signal from: (a) FNDs, (b) gold nanoparticles (GNPs), and (c) FND-5GNP under a single pulse of the excitation laser. (d) PA signal accumulated over 10 laser pulses from FNDs, GNPs, and FND-5GNP; where the 5 indicates that the particle ratio between the GNPs and FNDs is chosen to be 5:1; excitation wavelengths at 530 and $565 \mathrm{~nm}$, respectively. Reprinted with permission from ref. ${ }^{[63]}$ Copyright The Optical Society.

energy absorbing material, ${ }^{[62]}$ also the presence of the nanodiamond amplifies the photoacoustic signal of the gold. Several authors have shown that the energy transfer between ND and AuNP is able to increase the photoacoustic signal, allowing to

251 reduce the laser power engaged for the generation of the signal and then to improve the durability of the gold particles avoiding the dissolution due to long-time irradiation.

For this type of experiment, both $100 \mathrm{~nm}^{[63]}$ fluorescent nanodiamonds (FND) and detonation nanodiamonds of 4-5 nm 256 size were used. ${ }^{[51]}$ In the two cases, the authors observe a noticeable increase in the photoacoustic signal (PA) in experiments in-silicon and in-vivo, respectively.

In the case of FND, ${ }^{[63]}$ the PA signal is amplified even 30 times using a green light source $(530-560 \mathrm{~nm})$ which is reso-

261 nant with the absorption of the NV optical centers of the diamond (Figure 3).

Moreover, the authors highlight the quenching of the ND fluorescence speculating an energy transfer mechanism be- the rate and the quantity of the cell NPs uptake. Moreover, both 286 the label and therapeutic properties can be monitored together by confocal microscopy. Otherwise, compared to the researches previously described the fluorescence of the system seems not affected by the presence of gold and the authors did not detect any quenching of fluorescence. It is important to underline that 291 in this case the used NDs are not fluorescence itself but exhibit a broad fluorescence due to surface passivation with PEG.

AuNP-ND hybrid particles, prepared by a protein-mediated methodology developed by W. Liu et al., ${ }^{[61]}$ exhibit similar behavior. Despite the intrinsically fluorescent NDs used in this 296 work, the conjugation between the metals nanoparticles and the nanodiamonds takes place through a polymeric shell that probably inhibits any energy transfer between the two components. This system was tested as a marker for high-resolution cellular investigation in both optical imaging and transmission 301 electron microscopy (TEM). The authors report several advantages in optical microscopy in both the emission [excitation 


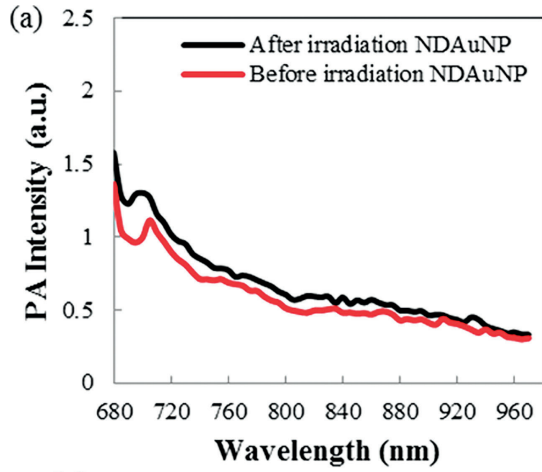

(b) NDAuNPs

0

30

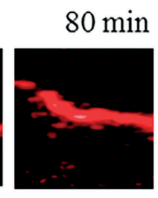

AuNPs
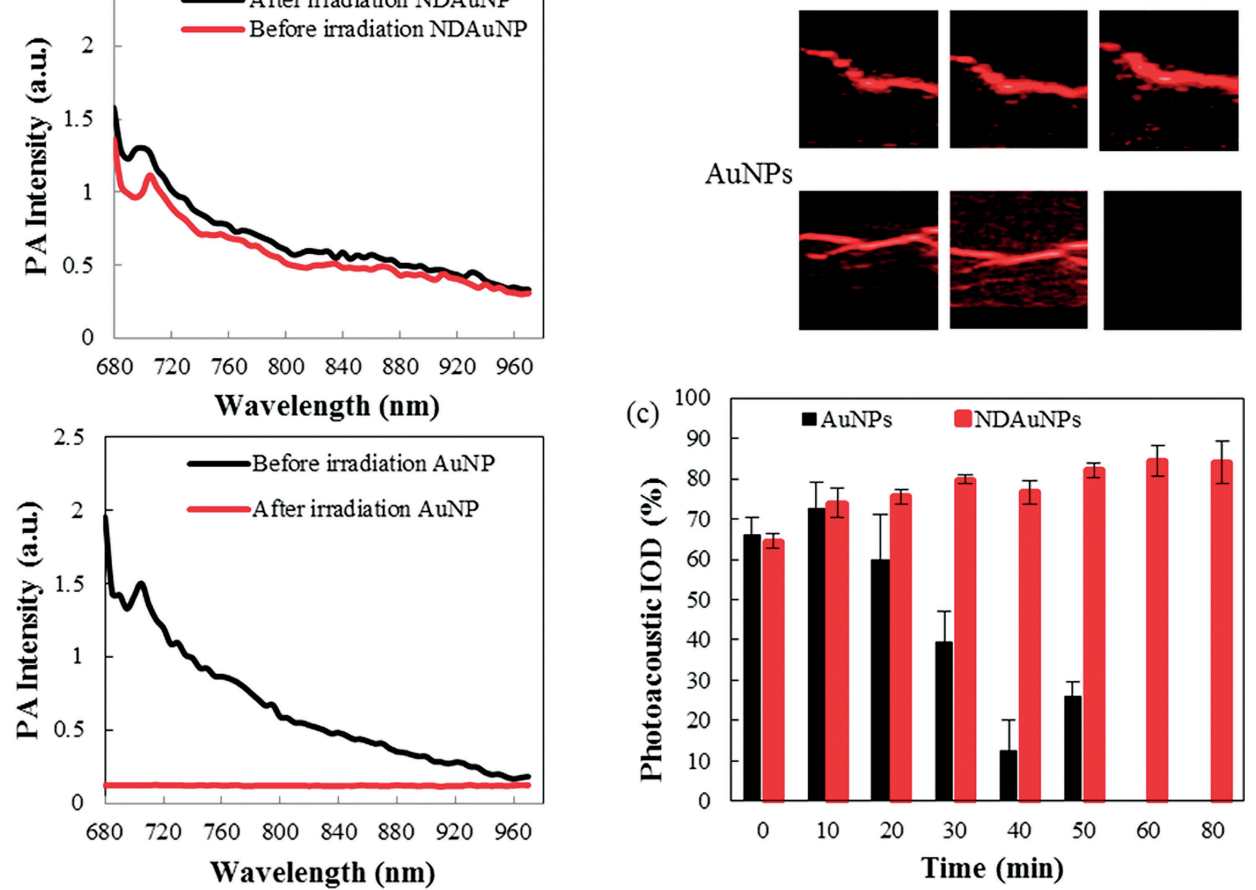

Figure 4. Photoacoustic spectra of AuNP-ND (named in the figure NDAuNP) and AuNPs before and after 80 min of laser irradiation. (b) Acquired PA images of AuNP-ND and AuNP at the respective times. (c) Photoacoustic intensity of AuNP-ND and AuNP (100 $\mu \mathrm{L}$ each) were injected into chicken breast muscle. Reprinted with permission from ref. ${ }^{[51]}$ Copyright 2017 American Chemical Society $((<=$ Author: Please check all copyright statements in the Figure captions, as some of them were corrected.))

source ( $\lambda$ ex) at $561 \mathrm{~nm}$ and emission wavelength at 575$718 \mathrm{~nm}$ spectral range] and scattering mode ( $\lambda$ ex $=458 \mathrm{~nm}$, 306 scattering wavelength at $450-470 \mathrm{~nm}$ spectral range) due to the photostable fluorescence originate from ND and localized surface plasmon resonance from gold respectively, Figure 5.
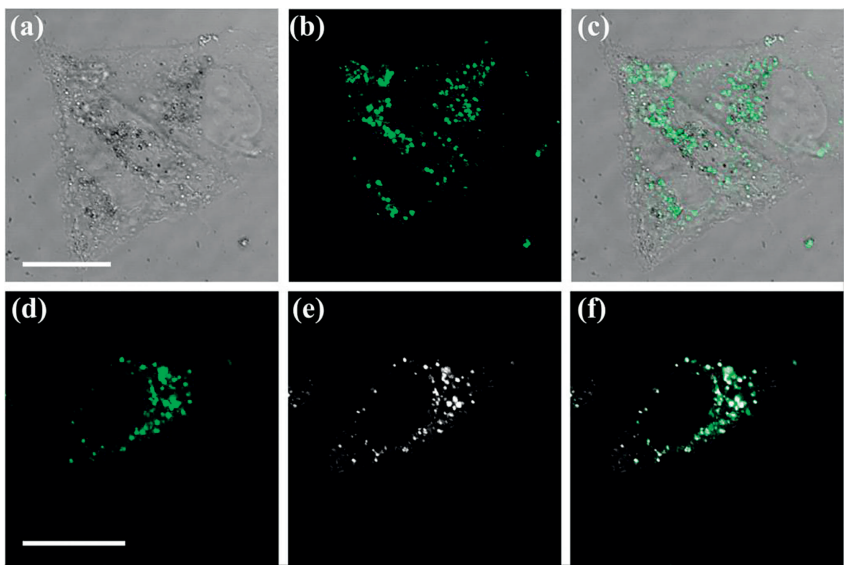

Figure 5. Optical imaging of A549 cells loaded with AuNP-ND (a) Bright field. (b) Fluorescence channel (c) Overlap of (a) and (b). Fluorescent and scattering imaging of AuNP-ND in A549 cells. (d) Fluorescence channel. (e) Scattering channel. (f) Overlap of (d) and (e). Scale bar: $20 \mu \mathrm{m}$. Reprinted with permission from ref. ${ }^{[61]}$ Copyright 2016 American Chemical Society.

Finally, the Au strongly improve the contrast in TEM images Figure 6. This allowed the authors to highlight an increased 311 number of vesicles in the cell, probably caused to the penetra- tion of sharp-edged diamonds through the endosomal membrane.
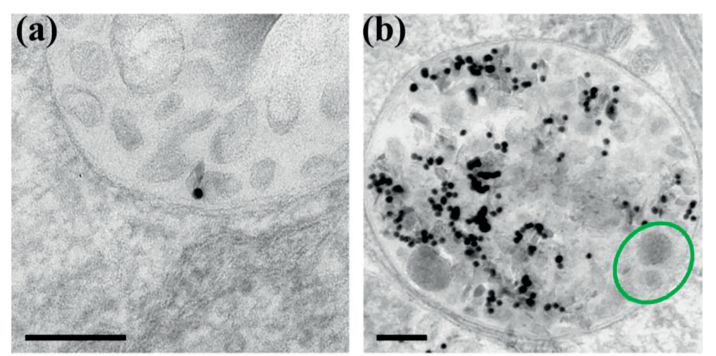

Figure 6. FND-Au cell slice observed by TEM based on the high-pressure freezing procedure. (a) Single FND-Au inside the cellular endosome. (b) FNDAu distributed in endosomes and cytosol. The green circle indicates luminal vesicles derived from the endosomal membrane penetration of the sharpedged FND-Au. Reprinted with permission from ref. ${ }^{[61]}$ Copyright 2016 American Chemical Society.

\subsection{SERS and Surface Plasmon Resonance Sensors}

Sensing and biosensing based on SERS require active substrates able to provide large enhancement factor and long-term stabil- 316 ity. Gold decorated nanodiamonds successfully used as SERS substrate shown excellent enhancement activity mainly based on charge transfer process. ${ }^{[51]}$

The charge transfer mechanism is particularly advantageous in all cases of fluorescent substrates, in fact, the charge or en- 321 ergy transfer turns off the fluorescence allowing the acquisition 
of the Raman signal. Furthermore, as in the case of the photoacoustic signal, diamond can easily dissipate the heat developed by the irradiated metal, increasing the durability of the SERS 326 substrate.

Recently these particles have been used for the molecular identification of foreign pigments on ancient supports.

An approach, based on integrated techniques and chemical analysis by SERS, made possible to identify the biological actor 331 (agent) responsible for specific purple-colored damage on ancient parchments solving a mystery long centuries. ${ }^{[64]}$

AuNP-ND hybrid systems can be used for drug delivery of hydrophobic substances too. Indeed nanodiamond allows the transport of insoluble substances in an aqueous environment,

336 avoiding the use of potentially toxic solvents or surfactants. ${ }^{[65]}$ An often-uncontrollable and/or neglected aspect in drug delivery is the monitoring of the integrity of the transported molecules that could undergo irreversible degradation or absorption on the substrate. It is possible to overcome this drawback

341 studying the adsorbed molecules by SERS and to monitor their structure and release on real-time by using AuNP-ND as drug delivery system. ${ }^{[65,66]}$

Nanodiamond conjugated gold nanoparticles were reported as an analytical colorimetric probe for the detection of Clen346 buterol hydrochloride (CLB) and $\mathrm{Cr}^{\mathrm{III}}$ ions both in ideal and real samples as lake water and urine. Several interference molecules and metal ions have been tested and did not affect the surface plasmon resonance (SPR) peak showing great selectively and sensitivity. ${ }^{[67]}$ The reported results seem impressive and deserve 351 further investigations.

\subsection{Catalysts}

AuNP-NDs shown catalytical activity in the hydroamination reaction of phenylacetylene with aniline, ${ }^{[53]}$ decomposition of aqueous solutions of hydrogen peroxide, ${ }^{[68]}$ and displayed per356 oxidase-like catalytic activity. ${ }^{[50]}$

The peroxidase-like catalytic activity was tested both for Au and Pt-decorated nanodiamond. Preliminary studies were performed on a diluted solution of $\mathrm{H}_{2} \mathrm{O}_{2}$ and then, in vitro, promoting reactive oxygen species (ROS) production by the addition

361 of rotenone. The obtained data show that the catalytic activity as antioxidant of $\mathrm{Au}$ and Pt-decorated nanodiamond is higher than that of glutathione, the most common compound employed as antioxidant. Finally considering both the antioxidant activity and biocompatibility the AuNP-ND is the material to 366 choose. ${ }^{[68]}$

AuNP-ND seems to exhibit oxidative catalytic activity following a ping-pong mechanism for multiple substrates. Moreover, as demonstrated by Min-Chul Kim et al., ${ }^{[50]}$ neither nanodiamonds nor gold nanoparticles alone used in this work exhibited

371 a peroxidase-like activity Figure $7 .^{[50]}$ To rationalize this claim the authors perform a deep investigation of the surface chemistry of all the used particles (ND, AuNPs, and AuNP-ND) by XPS. The results support the idea that the higher catalytic activity is related to the charge transfer from gold nanoparticles to $\mathrm{sp}^{2}$ 376 dangling bonds on ND surface. ${ }^{[50]}$

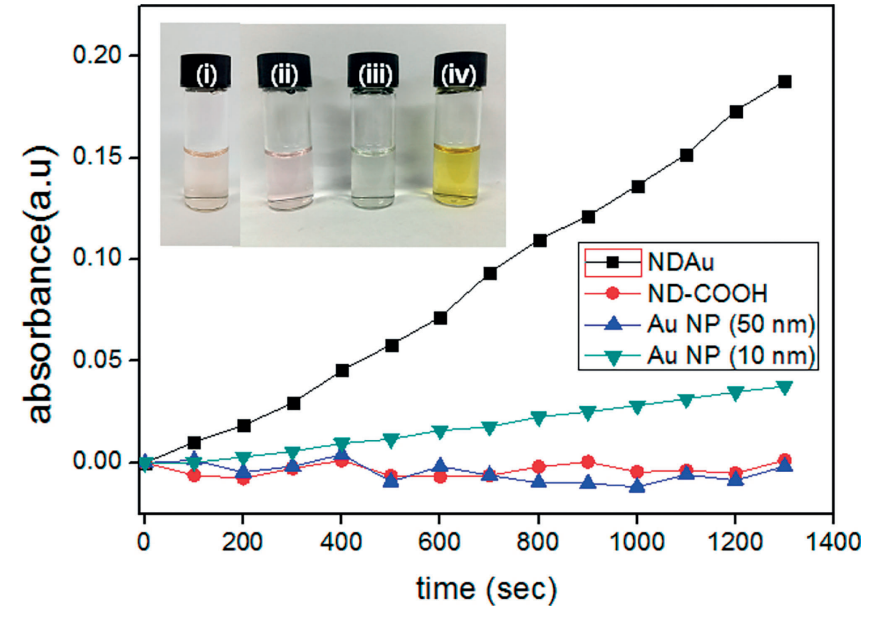

Figure 7. Progress of the catalytic oxidation reaction. The intensity of the UV absorbance peak of 2,3- diaminophenazine, the product of the oxidation of o-phenylenediamine, at $430 \mathrm{~nm}$ was monitored. Reprinted with permission from ref. ${ }^{[50]}$ Copyright 2016 American Chemical Society.

\section{New Prospects and Conclusion}

The AUNP-ND hybrid system has numerous advantages compared to the individual nanoparticles as they are.

In the case of direct synthesis of AuNP on ND surface, ND seems to anchor stably AuNP, preventing Au over-growth and 381 aggregation, without the use of potentially toxic surfactants normally used to synthesize AuNP alone. The technological issue in this case is the preparation of stable ND dispersion, on porpoise, numerous improvements have been made in recent years, and today, are available aqueous dispersions of nanodia- 386 mond with ND aggregates size ranging from $300 \mathrm{~nm}$ to $10 \mathrm{~nm}$, and single-digit ND dispersion too. ${ }^{[8]}$ These dispersions are generally without additives and are widely used in biological applications.

Conversely, in the case of AuNP-ND system obtained by 391 post-synthetic conjugation treatments, contaminations deriving from the synthesis of AuNP (surfactant and/or cupping agent) are always present together with a coating on ND surface that allows the conjugation between the two species. In this case, the system is more complicated, in term of chemical control 396 and stability, but shows the advantage of being easily modified by larger molecules and/or biomarkers.

Regardless of the preparation method of AuNP-ND, nanodiamond improves the heat energy transfer from gold to the medium, as a result, AuNP became more resistant to dissolution 401 under strong irradiation.

One of the most promising results in the use of gold nanodiamond hybrid systems is related to the coupling of their outstanding optoelectronic properties. As demonstrated energy absorption, photoacoustic and scattering signals can be greatly 406 enhanced using AuNP-ND hybrid compared to those of the component materials alone. The system retains local plasmonic properties triggering enhanced effect in Raman and in photoacoustic applications.

The optical properties deriving from the coupling of these 411 two species are already not completely clear and predictable, showing in some cases contradictory results. As a Matter of fact, 
shape and size of the particles, and the methodology used to conjugate them, determine the final properties of the hybrid 416 system. In general, the gold nanoparticles have the ability to quench the fluorescence of the nanodiamond when intimately conjugated to it. Despite this, using larger nanodiamond (in the range of $100 \mathrm{~nm}$ of diameter) and optimizing the distance between the surface plasmon and the optical center it was pos-

421 sible to obtain hybrid nanoparticles with an emission intensity of $30 \%$ lower to the nanodiamond alone. ${ }^{[54]}$

Nevertheless, they undoubtedly offer great potential for optical and photonic applications. ${ }^{[52,69-71]}$

AuNP-ND system has proved to be an excellent SERS plat426 form, able to penetrate cell and tissue and future research will cover its use both in vitro and in vivo studies.

The research related to sensors based on AuNP-ND systems is still in an embryonic phase. The first results demonstrate high sensitivity and selectivity, and further great results are expected

431 in the field of optical, electrochemical and electrochromic sensors. We believe with the further improvement of synthetic procedures, in term of control in size distribution and shape, the AuNP-ND nanoparticles will find more and more applications in biological and theranostics fields.

\section{Acknowledgments}

I will forever be thankful to Professor Emeritus Maria Letizia Terranova. She was and remains my best role model for a scientist and mentor. I'm thankful to Sara Politi for careful reading of this manuscript.

441 Keywords: Gold · Nanoparticles - Multifunctional materials · Nanodiamonds · Sensors

[1] V. P. Torchilin, Adv. Drug Delivery Rev. 2006, 58, 1532-1555.

[2] R. Hao, R. Xing, Z. Xu, Y. Hou, S. Gao, S. Sun, Adv. Mater. 2010, 22, 27292742.

446 [3] D.-E. Lee, H. Koo, I.-C. Sun, J. H. Ryu, K. Kim, I. C. Kwon, Chem. Soc. Rev. 2012, 41, 2656-2672.

[4] M. Motornov, Y. Roiter, I. Tokarev, S. Minko, Prog. Polym. Sci. 2010, 35, 174-211.

[5] M. A. Hayat, Colloidal Gold, Principles, Methods, and Applications, Vol. 1, Academic Press, San Diego, USA, 1989.

[6] M.-C. Daniel, D. Astruc, Chem. Rev. 2004, 104, 293-346.

[7] V. N. Mochalin, O. Shenderova, D. Ho, Y. Gogotsi, Nat. Nanotechnol. 2012, 7, 11-23.

[8] J.-C. Arnault (Ed.), Nanodiamonds Advanced Material Analysis, Properties 456 and Applications, Elsevier, 2017.

[9] A. Ruivo, C. Gomes, A. Lima, M. L. Botelho, R. Melo, A. Belchior, A. Pires de Matos, J. Cultural Heritage 2008, 9, e134-e137.

[10] S. Mahdihassan, Am. J. Chin. Med. 1985, 13, 93-108.

[11] P. K. Jain, X. Huang, I. H. El-Sayed, M. A. El-Sayed, Plasmonics 2007, 2, 461 107-118.

[12] M.-C. Daniel, D. Astruc, Chem. Rev. 2004, 104, 293-346 $\square((<=\square \square A u-$ thor: duplicate with ref.[6] घ)) $\square$.

[13] S. K. Ghosh, T. Pal, Chem. Rev. 2007, 107, 4797-4862.

[14] T. K. Sau, C. J. Murphy, J. Am. Chem. Soc. 2004, 126, 8648-8649.

466 [15] J. Zhou, J. Ralston, R. Sedev, D. A. Beattie, J. Colloid Interface Sci. 2009, $331,251-262$.

[16] N. Li, P. Zhao, D. Astruc, Angew. Chem. Int. Ed. 2014, 53, 1756-1789; Angew. Chem. 2014, 126, 1784.

[17] K. Saha, S. S. Agasti, C. Kim, X. Li, V. M. Rotello, Chem. Rev. 2012, 112, 471 2739-2779.
[18] W. Hou, S. B. Cronin, Adv. Funct. Mater. 2013, 23, 1612-1619.

[19] W. L. Barnes, A. Dereux, T. W. Ebbesen, Nature 2003, 424, 824-830.

[20] J. W. Kang, P. T. C. So, R. R. Dasari, D.-K. Lim, Nano Lett. 2015, 15, 17661772.

[21] C. Zong, M. Xu, L.-J. Xu, T. Wei, X. Ma, X.-S. Zheng, R. Hu, B. Ren, Chem. 476 Rev. 2018, 118, 4946-4980.

[22] V. V. Danilenko, Phys. Solid State 2004, 46, 595-599.

[23] G.-W. Yang, J.-B. Wang, Q.-X. Liu, J. Phys. Condens. Matter 1998, 10, 7923.

[24] S. Heyer, W. Janssen, S. Turner, Y.-G. Lu, W. S. Yeap, J. Verbeeck, A. Krueger, ACS Nano 2014, 8, 5757-5764.

[25] J. P. Boudou, P. A. Curmi, F. Jelezko, J. Wrachtrup, P. Aubert, M. Sennour G. Balasubramanian, R. Reuter, A. Thorel, E. Gaffet, Nanotechnology 2009, 20, 235602.

[26] Y. G. Gogotsi, K. G. Nickel, D. Bahloul-Hourlier, T. Merle-Mejean, G. E. Khomenko, K. P. Skjerlie, J. Mater. Chem. 1996, 6, 595-604.

[27] S. Korablov, K. Yokosawa, D. Korablov, K. Tohji, N. Yamasaki, Mater. Lett. 2006, 60, 3041-3044.

[28] T. L. Daulton, M. A. Kirk, R. S. Lewis, L. E. Rehn, Nucl. Instrum. Methods Phys. Res. Sect. B 2001, 175, 12-20.

[29] V. Gupta, P. Scharff, N. Miura, Mater. Lett. 2005, 59, 3259-3261.

[30] Galimov et al., Dokl. Phys. 2004, 49, 150-153.

[31] S.-J. Yu, M.-W. Kang, H.-C. Chang, K.-M. Chen, Y.-C. Yu, J. Am. Chem. Soc. 2005, 127, 17604-17605.

[32] W. W.-W. Hsiao, Y. Y. Hui, P.-C. Tsai, H.-C. Chang, Acc. Chem. Res. 2016, 49, 400-407.

[33] I. I. Vlasov, O. Shenderova, S. Turner, O. I. Lebedev, A. A. Basov, I. Sildos, M. Rähn, A. A. Shiryaev, G. Van Tendeloo, Small 2010, 6, 687-694.

[34] O. A. Shenderova, I. Vlasov, S. Turner, G. Van Tendeloo, S. B. Orlinskii, et al., J. Phys. Chem. C 2011, 115, 14014-14024.

[35] V. A. Shershulin, V. S. Sedov, A. Ermakova, U. Jantzen, L. Rogers, A. A. 501 Huhlina, E. G. Teverovskaya, V. G. Ralchenko, F. Jelezko, I. I. Vlasov, Phys. Status Solidi A 2015, 212, 2600-2605.

[36] H. Dong, B. Wen, R. Melnik, Sci. Rep. 2014, 4, 7037.

[37] M. A. Angadi, T. Watanabe, J. Appl. Phys. 2006, 99, 11430.

[38] M. L. Terranova, S. Orlanducci, M. Rossi, E. Tamburri, Nanoscale 2015, 7, 506 5094-5114.

[39] D. Zhu, L. Zhang, R. E. Ruther, R. J. Hamers, Nat. Mater. 2013, 12, 836841.

[40] M. Kurzyp, H. A. Girard, Y. Cheref, E. Brun, C. Sicard-Roselli, S. Saada, J.-C. Arnault, Chem. Commun. 2017, 53, 1237-1240.

[41] R. Grall, H. Girard, L. Saad, T. Petit, C. Gesset, M. Combis-Schlumberger, V. Paget, J. Delic, J.-C. Arnault, S. Chevillard, Biomaterials 2015, 61, 290 298.

[42] A. Krueger, D. Lang, Adv. Funct. Mater. 2012, 22, 890-906.

[43] A. Krueger, Chem. Eur. J. 2008, 14, 1382-1390.

[44] L. Lai, A. S. Barnard, J. Nanosci. Nanotechnol. 2015, 15, 989-999.

[45] H. Moche, V. Paget, D. Chevalier, E. Lorge, N. Claude, H. A. Girard, J. C. Arnault, S. Chevillard, F. Nesslany, J. Appl. Toxicol. 2017, 37, 954-961.

[46] N. Mohan, C.-S. Chen, H.-H. Hsieh, Y.-C. Wu, H.-C. Chang, Nano Lett. 2010, 10, 3692-3699.

[47] V. Vaijayanthimala, P.-Y. Cheng, S.-H. Yeh, K.-K. Liu, C.-H. Hsiao, J.-I. Chao, H.-C. Chang, Biomaterials 2012, 33, 7794-7802.

[48] A. M. Schrand, H. Huang, C. Carlson, J. J. Schlager, E. Ōsawa, S. M. Hussain, L. Dai, J. Phys. Chem. B 2007, 111, 2-7.

[49] D. Ho, C.-H. K. Wang, E. K.-H. Chow, Sci. Adv. 2015, 1, e1500439.

[50] M.-C. Kim, D. Lee, S. H. Jeong, S.-Y. Lee, E. Kang, ACS Appl. Mater. Interfaces 2016, 8, 34317-34326.

[51] D. Lee, E.-J. Park, S.-E. Lee, S. H. Jeong, J. Y. Lee, E. Kang, ACS Sustainable Chem. Eng. 2017, 5, 8284-8293.

[52] D. Potamianos, I. Papadakis, E. Kakkava, A. B. Bourlinos, G. Trivizas, R. 531 Zboril, S. Couris, J. Phys. Chem. C 2015, 119, 24614-24620.

[53] A. Seral-Ascaso, A. Luquin, M. Jesús Lázaro, G. F. de la Fuente, M. Laguna, E. Muñoza, Appl. Catal. A 2013, 456, 88-95.

[54] L. Minati, C. L. Cheng, Y. C. Lin, J. Hees, G. Lewes-Malandrakis, C. E. Nebel, F. Benetti, C. Migliaresi, G. Speranza, Dia. Relat. Mater. 2015, 53, 23-28. 536

[55] S. Orlanducci, I. Cianchetta, E. Tamburri, M. L. Terranova, M. C. Cassani, R. Matassa, M. Rossi, MRS Proc. 2012, 1452, mrss12.

[56] R. Martín, M. Álvaro, J. R. Herance, H. García, ACS Nano 2010, 4, 65-74.

[57] R. Matassa, S. Orlanducci, G. Reina, M. C. Cassani, D. Passeri, M. L. Terranova, M. Rossi, Sci. Rep. 2016, 6, 31163. 
[58] B. Ballarin, L. Busetto, M. C. Cassani, C. Femoni, Inorg. Chim. Acta 2010, 363, 2055-2064

[59] Y. Zeng, W. Liu, Z. Wang, S. Singamaneni, R. Wang, Langmuir 2018, 34, 4036-4042.

546 [60] L.-C. Cheng, H. M. Chen, T.-C. Lai, Y.-C. Chan, R.-S. Liu, J. C. Sung, M. Hsiao, C.-H. Chen, L.-J. Her, D. P. Tsai, Nanoscale 2013, 5, 3931-3940.

[61] W. Liu, B. Naydenov, S. Chakrabortty, B. Wuensch, K. Hübner, S. Ritz, H. Cölfen, H. Barth, K. Koynov, H. Qi, R. Leiter, R. Reuter, J. Wrachtrup, F. Boldt, J. Scheuer, U. Kaiser, M. Sison, T. Lasser, P. Tinnefeld, F. Jelezko, P. Walther, Y. Wu, T. Weil, Nano Lett. 2016, 16, 6236-6244.

[62] Y. Toumia, F. Domenici, S. Orlanducci, F. Mura, D. Grishenkov, P. Trochet, S. Lacerenza, F. Bordi, G. Paradossi, ACS Appl. Mater. Interfaces 2016, 8, $16465-16475$.

[63] B. Zhang, C.-Y. Fang, C.-C. Chang, R. Peterson, S. Maswadi, R. D. Glickman, H.-C. Chang, J. Y. Ye, Biomed. Opt. Express 2012, 3, 1662-1669.

[64] L. Migliore, M. C. Thaller, G. Vendittozzi, A. Yazmine Mejia, F. Mercuri, S. Orlanducci, A. Rubechini, Sci. Rep. 2017, 7, 9521.
[65] A. Gismondi, G. Reina, S. Orlanducci, F. Mizzonia, S. Gay, M. L. Terranova, A. Canini, Biomaterials 2015, 36, 22-35.

[66] G. Reina, S. Orlanducci, C. Cairone, E. Tamburri, S. Lenti, I. Cianchetta, M. 561 Rossi, M. L. Terranova, J. Nanosci. Nanotechnol. 2015, 15, 1022-1029.

[67] M. Shellaiah, T. Simon, P. Venkatesan, K. W. Sun, F.-H. Ko, S.-P. Wu, Microchim. Acta 2018, 185, 74.

[68] R. Martın, C. Menchon, N. Apostolova, V. M. Victor, M. Alvaro, J. R. I. Herance, H. García, Acs Nano 2010, 4, 6957-6965.

[69] G. Reina, S. Orlanducci, E. Tamburri, R. Matassa, M. Rossi, M. L. Terranova, Phys. Status Solidi C 2016, 1-7.

[70] M. Barth, S. Schietinger, T. Schröder, T. Aichele, O. Benson, J. Lumin. 2010, 130, 1628-1634.

[71] J. Zhao, Y. Cheng, H. Shen, Y. Y. Hui, T. Wen, H.-C. Chang, Q. Gong, G. Lu, 571 Sci. Rep. 2018, 8, 3605.

Received: June 23, 2018 
Gold Nanostructures in Medicine

576 S. Orlanducci* $1-9$

(D) Gold-Decorated Nanodiamonds: ${ }_{581}$ Powerful Multifunctional Materials for Sensing, Imaging, Diagnostics, and Therapy

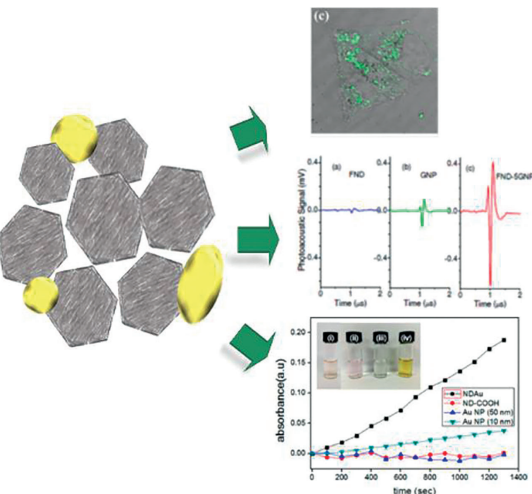

Gold nanoparticle nanodiamond (AuNP-ND) hybrids are a powerful multifunctional material for biological and theranostic applications. The shape and size of the particles and the methodology used to conjugate them determine the final properties of the system. Energy absorption, SERS, photoacoustic, scattering signals, and finally catalytic and sensing properties can be enhanced by these hybrids.

Review article by Silvia Orlanducci @UTORV on the application of gold nanoparticle nanodiamond hybrids as multi591 functional materials for biological and theranostic applications

Share your work on social media! The European Journal of Inorganic Chemistry has added Twitter as a means to promote your article. Twitter is an online microblogging service that enables its users to send and read text-based messages of up to 140 characters, known as "tweets". Please check the pre-written tweet in the galley proofs for accuracy. Should you or your institute have a Twitter account, please let us know the appropriate username (i.e., @accountname), and we will do 596 our best to include this information in the tweet. This tweet will be posted to the journal's Twitter account @EurJIC (follow us!) upon online publication of your article, and we recommend you to repost ("retweet") it to alert other researchers about your publication.

Authors: Please check that the ORCID identifiers listed below are correct. We encourage all authors to provide an ORCID identifier for each coauthor. ORCID is a registry that provides researchers with a unique digital identifier. Some funding 601 agencies recommend or even require the inclusion of ORCID IDs in all published articles, and authors should consult their funding agency guidelines for details. Registration is easy and free; for further information, see http://orcid.org/.

Silvia Orlanducci http://orcid.org/0000-0002-3261-933X*

DOI: 10.1002/ejic.201800793 